\title{
Efficacy of tenofovir-based rescue therapy for chronic hepatitis B patients with resistance to lamivudine and entecavir
}

\author{
Hee-Jeong Jeon', Seok Won Jung', Neung Hwa Park ${ }^{1,2}$, Yujin Yang', Jin-Hee Noh', Jae-Sung Ahn', Hyung Rae Kim', \\ Jae Ho Lee ${ }^{3}$, and Jung Woo Shin \\ Departments of Internal Medicine, ${ }^{2}$ Biomedical Research Center and ${ }^{3}$ Anesthesiology and Pain Medicine, Ulsan University Hospital, \\ University of Ulsan College of Medicine, Ulsan, Korea
}

Background/Aims: Tenofovir disoproxil fumarate (TDF) monotherapy for 48 weeks provided a virological response comparable to that of TDF and entecavir (ETV) combination therapy in patients infected with ETV-resistant hepatitis B virus $(\mathrm{HBV})$. Little long-term data in routine clinical practice are available regarding the optimal treatment of patients with ETV-resistant HBV.

Methods: We investigated the long-term antiviral efficacy of combination therapy of TDF+lamivudine (LAM) or TDF+ETV compared to that of TDF monotherapy in 73 patients with resistance to both LAM and ETV.

Results: Patients were treated with TDF monotherapy $(n=12)$, TDF+LAM $(n=19)$, or TDF+ETV $(n=42)$ for more than 6 months. The median duration of TDF-based rescue therapy was 37 months. Virologic response (VR) was found in 63 patients (86.3\%). The rates of VR among the three groups (TDF monotherapy, TDF+LAM, and TDF+ETV) were not statistically different (log-rank $P=0.200)$ at 12 months $(59.3 \%, 78.9 \%$, and $51.8 \%$, respectively) or at 24 months (88.4\%, $94.7 \%$, and 84.2\%). In addition, treatment efficacy of TDF-based combination or TDF monotherapy was not statistically different with ETV-resistant strains or exposure to other antiviral agents. In multivariate analysis, only lower baseline HBV DNA level was an independent predictor for VR (hazard ratio, 0.723 ; 95\% confidence interval, 0.627-0.834; $P<0.001$ ).

Conclusions: TDF monotherapy was as effective as combination therapy of TDF+LAM or TDF+ETV in maintaining long-term viral suppression in chronic hepatitis B patients with resistance to both LAM and ETV. HBV DNA level at the start of TDF rescue therapy was the only independent predictor of subsequent VR. (Clin Mol Hepatol 2017;23:230-238)

Keywords: Tenofovir; Resistance; Lamivudine; Entecavir; Chronic hepatitis B

\section{INTRODUCTION}

Hepatitis B virus (HBV) infection is a serious public health problem worldwide, with an estimated 2 billion infections, and a ma- jor cause of chronic hepatitis, cirrhosis, and hepatocellular carcinoma (HCC). ${ }^{1}$ Higher levels of HBV DNA are associated with an increased risk for HCC and cirrhosis in patients with chronic hepatitis $B(C H B){ }^{2-5}$ By contrast, reducing HBV DNA concentrations to

\footnotetext{
Abbreviations:

ADV, adefovir dipivoxil; AE, adverse event; ALT, alanine transaminase; ANOVA, analysis of variance; $A U R O C$, area under the ROC curve; $C H B$, chronic hepatitis $\mathrm{B} ; \mathrm{Cl}$, confidence interval; ETV, entecavir; $\mathrm{HBeAg}$, hepatitis B e antigen; $\mathrm{HBsAg}$, hepatitis B surface antigen; HBV, hepatitis B virus; HCC, hepatocellular carcinoma; HIV, human immunodeficiency virus; LAM, lamivudine; MDR, multi-drug resistance; OR, odds ratio, PCR, polymerase chain reaction; PVR, partial virologic response; RFMP, restriction fragment mass polymorphism; ROC, receiver operating characteristic; SAE, serious adverse event; TDF, tenofovir disoproxil fumarate; VBT, virologic breakthrough; VR, virologic response
}

Corresponding author : Neung Hwa Park

Department of Internal Medicine, Ulsan University Hospital, 877 Bangeojinsunhwando-ro, Dong-gu, Ulsan 44033, Korea

Tel: +82-52-250-7029, Fax: +82-52-250-7048

E-mail: nhpark@uuh.ulsan.kr

http://orcid.org/0000-0002-5648-9189 
Hee-Jeong Jeon, et al.

TDF in CHB patients with LAM and ETV resistance

very low or undetectable levels through antiviral therapy is associated with reduced risk of mortality and/or HCC. ${ }^{4-6}$

Nucleos(t)ide analogues (NUCs) as an important class of antiviral drugs have changed the treatment paradigm and prognosis of CHB. Lamivudine (LAM), the first oral antiviral agent approved to treat $\mathrm{HBV}$, is safe and well tolerated, even in patients with decompensated liver cirrhosis. ${ }^{7}$ However, the long-term use of LAM inevitably leads to the development of resistant HBV mutants. ${ }^{8}$ Earlier treatment guidelines based on insufficient clinical experiences have recommended the use of entecavir (ETV) as one of the treatment options for CHB patients with LAM resistance. ${ }^{9,10}$ However, in patients with pre-existing LAM-resistance, the rate of ETV resistance increases up to $51 \%$ after 5 years of sequential ETV treatment. ${ }^{11}$ Emergence of NUCs-resistant HBV usually results in attenuated viral suppression and disease progression which may lead to significant clinical deterioration. ${ }^{12,13}$

Tenofovir disoproxil fumarate (TDF) is a potent nucleotide analog recommended and used once-daily as a first line therapy for CHB patients. ${ }^{14,15}$ TDF is also recommended for patients who have developed resistance to LAM, ETV, or telbivudine. In addition, in vitro studies suggest that ETV-resistant HBV mutants are susceptible to TDF. ${ }^{16}$ Several case reports and retrospective cohort studies also showed the clinical efficacy of TDF in ETV-resistant or ETV-refractory patients. ${ }^{17-20}$ In a recent randomized controlled study, TDF mono-rescue therapy for 48 weeks induced a virologic response comparable to that of TDF and ETV combination therapy in patients infected with ETV-resistant HBV. ${ }^{21}$ In another study, the TDF group also showed a high virologic response rate comparable to that of the TDF+ETV group, but the duration of follow-up was relatively short (a median 18 months). ${ }^{22}$ Therefore, the objective of this study was to investigate the long-term outcomes of TDF based rescue therapy in CHB patients with resistance to both LAM and ETV in routine clinical practice. In addition, the efficacy of TDF combination therapy (with LAM or ETV) was compared to that of TDF monotherapy.

\section{PATIENTS AND METHODS}

\section{Patient population}

Electronic medical records of CHB patients with ETV resistance in addition to prior LAM resistance were reviewed. Patients were treated with sequential ETV monotherapy due to genotypic LAM resistance. A total of 73 patients were treated with TDF alone or combination (with LAM or ETV) by clinician or patient choice from December 2012 to November 2015. All patients had hepatitis B surface antigen (HBsAg). Serum HBV DNA level was documented for at least 6 months before the start of LAM therapy. Patients with impaired renal function (serum creatinine $>1.5 \mathrm{mg} / \mathrm{dL}$ ), autoimmune hepatitis, or antibodies against hepatitis $C$ virus or human immunodeficiency virus (HIV) were excluded from this study. Additional criteria for exclusion were: pregnancy, lactation, and alcohol abuse (>40 g/d ethanol). Diagnoses of chronic hepatitis and liver cirrhosis were based on liver biopsy features or on clinical, laboratory, and ultrasound data if liver biopsy data were unavailable. Written informed consents were obtained from all of patients participating in this study. This research was approved by the Institutional Review Board at the Ulsan University Hospital (UUH 2016-09-002, approved on Sep. 20, 2016).

\section{Laboratory measurements}

Liver and kidney function tests were performed every three months during TDF based rescue treatment. HBV DNA levels were quantified using COBAS TaqMan HBV test (Roche, Branchburg, $\mathrm{NJ}$, USA) with a lower detection limit of $12 \mathrm{IU} / \mathrm{mL}$ (60 copies $/ \mathrm{mL}$ ). Genotypic resistance to ETV was defined as the emergence of ETV resistance mutations in the presence of lamivudine resistance substitutions M204I/V \pm L180M. When a viral breakthrough had developed before TDF-based rescue therapy, we tested a restriction fragment mass polymorphism (RFMP; Genematrix, Youngin, Korea) to identify LAM (rt180, rt204), ADV (rt181, rt236), and ETV (rt173, rt184, rt202, rt250). The specific HBV genotypes were identified using polymerase chain reaction-restriction fragment length polymorphism (PCR-RFLP) of the surface gene of HBV genome. The two fragments of the HBV genome between the nucleotide positions 2823 and 2845 and 61 and 80 were amplified by PCR. PCR products were treated with restriction enzymes. Patients underwent surveillance for HCC every 6 months with serial abdominal ultrasound and serum $\alpha$-fetoprotein measurements.

\section{Definitions}

A virologic response (VR) was defined as the absence of serum HBV DNA by PCR assay $(<12 \mathrm{IU} / \mathrm{mL})$ on two consecutive measurements during TDF treatment. Hepatitis B envelop antigen ( $\mathrm{HBeAg}$ ) seroconversion was defined as the loss of HBeAg accompanied by the detection of anti-HBe. Partial virologic response (PVR) was defined as a decrease in HBV DNA of more than $1 \log _{10} \mathrm{IU} / \mathrm{mL}$ but 
with detectable HBV DNA after 6 months of antiviral therapy. Virologic breakthrough (VBT) was defined as a $>1 \log _{10} \mathrm{IU} / \mathrm{mL}$ increase in serum HBV DNA from the nadir on two consecutive measurements or in the last available measurement. Safety and tolerability were evaluated by the occurrence of adverse events (AEs), serious AEs (SAEs), laboratory abnormalities, discontinuation of the study drug due to AEs, or death. Specific markers of renal abnormalities included confirmed (defined as two consecutive visits) increase in serum creatinine of at least $0.5 \mathrm{mg} / \mathrm{dL}$ above the baseline value, serum phosphorus values $<2 \mathrm{mg} / \mathrm{dL}$, and creatinine clearance $<50 \mathrm{~mL} / \mathrm{min}$.

\section{Statistical analysis}

Serum HBV DNA (IU/mL) levels were logarithmically transformed for statistical analysis. Continuous variables were compared using the one-way analysis of variance (ANOVA). Categorical variables were compared using the $\chi^{2}$ test. The cumulative probability rates of clinical outcomes were calculated using Kaplan-Meier method. To identify the factors predictive of outcome among the baseline variables, the variables for clinical outcomes were compared using the $\chi^{2}$ test or univariate logistic regression. Variables with a $P$-value of 0.1 or less in the univariate analyses were included in a Cox step-wise multivariate regression analysis using forward selection. To determine predictive value of baseline serum HBV DNA level, the hazard ratio (HR) and receiver operating characteristic (ROC) curves with the overall accuracy assessed by the area under the curve (AUROC) were used for the prediction of VR for TDF treatment. The AUROCs and their corresponding 95\% confidence intervals (Cls) were measured to assess the degree of discrimination of the variables. All data were analyzed using Statistical Package for the Social Sciences software for Windows (version 21.0; SPSS Inc., Chicago, IL, USA). In all cases, a two-tailed $P$-value less than 0.05 was considered statistically significant.

\section{RESULTS}

\section{Overall clinical outcomes of TDF based therapy}

The baseline characteristics of the 73 CHB patients are summarized in Table 1. All patients were genotype C2. All patients had been treated with LAM as a first line oral antiviral agent followed by ETV treatment for LAM resistance. The median duration of LAM therapy was 32 months (range, 15-144 months). The median
Table 1. The baseline characteristics of the studied patients $(n=73)$

\begin{tabular}{lc}
\hline & Value \\
\hline Age (years) & $53.0(27-79)$ \\
\hline Gender (male/female) & $54 / 19$ \\
\hline Diagnosis (chronic hepatitis/cirrhosis) & $37 / 36$ \\
AST (IU/L) & $29(13-425)$ \\
\hline ALT (IU/L) & $32.0(8-589)$ \\
HBV DNA (log 10 IU/mL) & $4.11(1.42-8.23)$ \\
HBeAg positivity (n, \%) & $63(86.3)$ \\
\hline Duration of LAM therapy (months) & $32(15-144)$ \\
\hline Duration of ETV therapy (months) & $26.5(20-70)$ \\
Duration of TDF-based rescue therapy (months) & $37(6-45)$ \\
ETV resistant mutations (+M204I/V \pm L180M) & \\
\hline 169SMT,184IALT, 202G, 250LV,184LV 202G & $7,23,22,7,5$ \\
\hline
\end{tabular}

Continuous variables are expressed as medians with range.

AST, aspartate transaminase; ALT, alanine transaminase; HBV, hepatitis $B$ virus; HBeAg, hepatitis B e antigen; LAM, lamivudine; ETV, entecavir; TDF, tenofovir disoproxil fumarate.

duration of ETV treatment prior to TDF based rescue therapy was 26.5 months (range, 20-70 months). The known genotypic ETV mutations, 169SMT, 184IALT, 202G, 250LV, and 184LV 202G in the presence of $L A M$ resistance substitutions M204I/ $\pm \pm L 180 M$ were detected in $8,28,21,7$, and 9 patients, respectively. Patients were treated with TDF based rescue therapy for more than 6 months. Of the 73 patients, 42 received TDF+ETV combination therapy, 19 received TDF+LAM combination therapy, and 12 had TDF monotherapy. The median duration of TDF based rescue therapy was 37 months (range, 6 to 45 months). Of the 73 patients, 36 (49.5\%) had liver cirrhosis, 63 (86.3\%) were HBeAg positive. Twenty-three (31.5\%) patients had abnormal alanine transaminase (ALT) levels. All patients had persistent HBV viremia prior to TDF based rescue therapy. The median HBV DNA level prior to TDF based treatment was $4.11 \log _{10} \mathrm{IU} / \mathrm{mL}$ (range, 1.42-8.23 $\log _{10} \mathrm{IU} /$ $\mathrm{mL}$ ). Among the 23 patients with elevated ALT levels at baseline, 14 (60.9\%) patients had ALT normalization during TDF based rescue treatment. During TDF based rescue therapy, VR was achieved in 63 patients (86.3\%). The cumulative rates of VR at 12 and 24 months were $63.3 \%$ and $87.1 \%$, respectively. During TDF-based rescue therapy, 30 (41.1\%) had PVR. Among the $63 \mathrm{HBeAg-posi-}$ tive patients, 6 (9.5\%) achieved HBeAg seroconversion during TDF based treatment. However, no patient lost serum HBsAg during treatment. During the follow-up visits, 7 patients (9.6\%) were found to be non-adherent to TDF therapy (determined by review of medical and pharmacy records). Among these patients, 2 (2.7\%) 
Table 2. The baseline characteristics of the TDF monotherapy, TDF + LAM, and TDF + ETV treatment groups

\begin{tabular}{|c|c|c|c|c|}
\hline & $\begin{array}{c}\text { TDF } \\
(n=12)\end{array}$ & $\begin{array}{c}\text { TDF+LAM } \\
(n=19)\end{array}$ & $\begin{array}{c}\text { TDF+ETV } \\
(n=42)\end{array}$ & $P$-value \\
\hline Age (years) & $55.5 \pm 13.4$ & $54.8 \pm 10.5$ & $52.6 \pm 9.4$ & 0.592 \\
\hline Gender (male/female) & $8 / 4$ & $13 / 6$ & $33 / 9$ & 0.577 \\
\hline Diagnosis (chronic hepatitis/cirrhosis) & $7 / 5$ & $10 / 9$ & $20 / 22$ & 0.792 \\
\hline AST (IU/L) & $41.7 \pm 36.0$ & $26.7 \pm 6.8$ & $51.4 \pm 68.2$ & 0.263 \\
\hline $\operatorname{ALT}(I U / L)$ & $48.8 \pm 50.1$ & $33.1 \pm 13.5$ & $61.8 \pm 95.3$ & 0.393 \\
\hline HBV DNA $\left(\log _{10} I \mathrm{I} / \mathrm{mL}\right)$ & $4.10 \pm 2.80$ & $3.20 \pm 1.03$ & $4.69 \pm 1.89$ & 0.021 \\
\hline HBeAg positivity (n, \%) & $8(66.7)$ & $17(89.5)$ & $38(90.5)$ & 0.096 \\
\hline Duration of LAM therapy (months) & $32.9 \pm 29.7$ & $40.5 \pm 21.3$ & $31.5 \pm 29.3$ & 0.518 \\
\hline Duration of ETV therapy (months) & $23.4 \pm 20.1$ & $26.7 \pm 12.0$ & $28.1 \pm 13.4$ & 0.613 \\
\hline Duration of TDF therapy (months) & $25.8 \pm 14.4$ & $38.2 \pm 1.3$ & $33.6 \pm 9.8$ & 0.003 \\
\hline
\end{tabular}

Continuous variables are expressed as means \pm standard deviations.

TDF, tenofovir disoproxil fumarate; ETV, entecavir; LAM, lamivudine; AST, aspartate transaminase; ALT, alanine transaminase; HBV, hepatitis B virus; HBeAg, hepatitis B e antigen.

experienced VBT (increase in HBV DNA by $1 \log _{10} \mathrm{IU} / \mathrm{mL}$ at $9-12$ months of treatment). These 2 patients with VBT responded well to TDF rescue therapy after continuation of treatment.

\section{Comparison of VR among TDF alone, TDF+LAM, and TDF+ETV therapy}

To define whether there was any difference in the rate of $V R$ in TDF alone, TDF+LAM, or TDF+ETV therapy, VR rates were compared according to the variables using a log-rank test. The baseline characteristics of the TDF monotherapy $(n=12), T D F+L A M$ $(n=19)$, and TDF+ETV $(n=42)$ treatment groups are shown in Table 2. At baseline, there was no significant difference among the three groups except HBV DNA levels at the start of TDF based rescue therapy (4.10 \pm 2.80 in TDF alone, $3.20 \pm 1.03$ in TDF+LAM, and $4.69 \pm 1.89$ in TDF+ETV therapy, $P=0.021$ ) and the duration of TDF rescue treatment ( $25.8 \pm 14.4$ months in TDF alone, $38.2 \pm 13.0$ months in TDF+LAM, 33.6 \pm 9.8 months in TDF+ETV therapy, $P=0.003)$. The mean reduction rates of HBV DNA level during 12 months from baseline are shown in Fig. 1. The mean reduction in serum HBV DNA concentration was not significantly different among the three groups. Overall clinical outcomes among the three treatment groups are summarized in Table 3. The rates of VR among the three groups (TDF monotherapy, TDF+LAM, and TDF+ETV) were not statistically different (Fig. 2; log-rank $P=0.200)$ at 12 months $(59.3 \%, 78.9 \%$, and $51.8 \%$, respectively) or at 24 months $(88.4 \%, 94.7 \%$, and $84.2 \%$, respectively). Additionally, the proportion of patients that achieved a normalized ALT

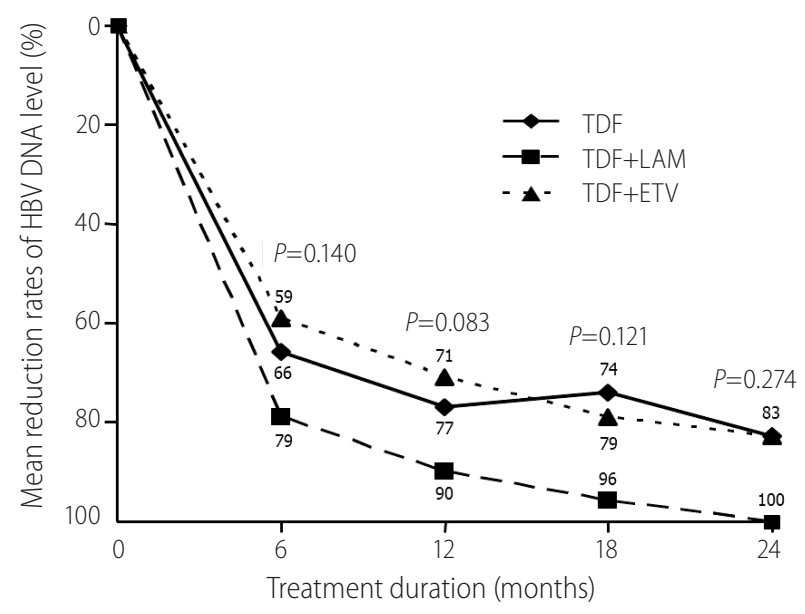

Figure 1. Mean reduction rates of HBV DNA level from baseline between TDF monotherapy, TDF+LAM, and TDF+ETV combination therapy groups. The mean reduction in serum HBV DNA concentration was not significantly different among the three treatment groups. HBV, hepatitis B virus; TDF, tenofovir disoproxil fumarate; ETV, entecavir; LAM, lamivudine.

level was also similar between the groups.

Of the 73 patients, 36 (the presence of ETV+ADV salvage treatment) received combination therapy of ETV+ADV as salvage therapy for LAM and ETV resistance prior to TDF based rescue therapy. The remaining 37 patients (the absence of ETV+ADV salvage treatment) only received ETV monotherapy prior to TDF based rescue therapy. The presence of ETV+ADV salvage treatment group had significantly lower baseline AST, ALT $(P<0.05$, respectively), and HBV DNA $(P<0.001)$ levels than the absence of 
Table 3. Overall clinical outcomes in the TDF alone, TDF+LAM, and TDF+ETV therapy groups

\begin{tabular}{lcccc}
\hline & $\begin{array}{c}\text { TDF } \\
(\mathbf{n}=\mathbf{1 2})\end{array}$ & $\begin{array}{c}\text { TDF+LAM } \\
(\mathbf{n}=\mathbf{1 9})\end{array}$ & $\begin{array}{c}\text { TDF+ETV } \\
(\mathbf{n}=\mathbf{4 2})\end{array}$ & $\boldsymbol{P}$-value \\
\hline HBeAg seroconversion $(\mathrm{n}=64,[\mathrm{n}, \%])$ & $0 / 8(0)$ & $2 / 17(11.8)$ & $4 / 39(10.3)$ & 0.161 \\
VR $(n, \%)$ & $9 / 12(75.0)$ & $19 / 19(100)$ & $35 / 42(83.3)$ & 0.099 \\
VBT $(n, \%)$ & $1(8.3)$ & $0(0)$ & $1(2.4)$ & 0.222 \\
PVR $(n, \%)$ & $5(41.7 \%)$ & $5(26.3)$ & $20(47.6)$ & 0.293 \\
\hline ALT normalization $(n=23,[n, \%])$ & $2 / 4(50)$ & $2 / 3(66.7)$ & $10 / 16(62.5)$ & 0.879 \\
\hline
\end{tabular}

Values are presented as $n(\%)$ unless otherwise indicated.

TDF, tenofovir disoproxil fumarate; ETV, entecavir; LAM, lamivudine; HBeAg, hepatitis B e antigen; VR, virologic response; VBT, virological breakthrough; PVR, partial virologic response; ALT, alanine transaminase.

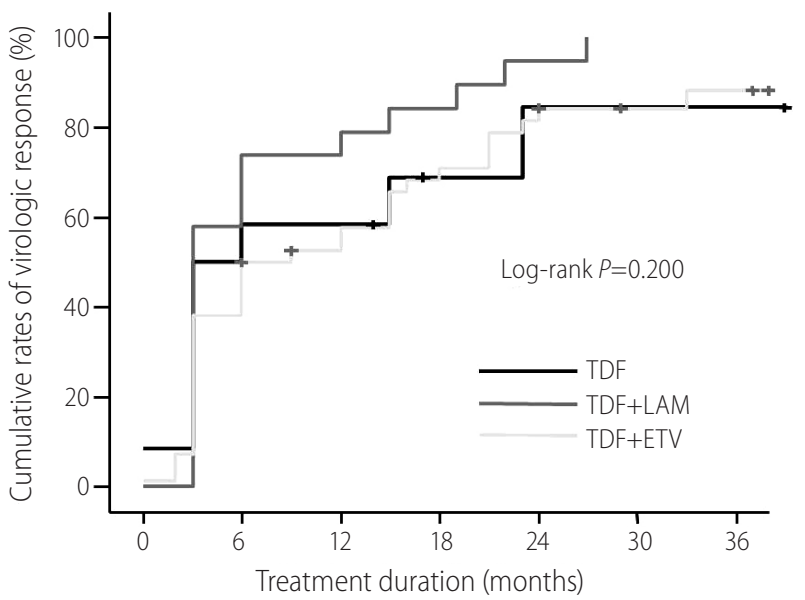

Figure 2. Cumulative rates of VR according to TDF rescue therapy. The rates of $V R$ among the three groups (TDF monotherapy, TDF+LAM, and TDF+ETV) were not statistically significant at 12 months (59.3\%, 78.9\%, and $51.8 \%$, respectively) or at 24 months (88.4\%, 94.7\%, and 84.2\%). VR, virologic response; TDF, tenofovir disoproxil fumarate; ETV, entecavir; LAM, lamivudine.

ETV+ADV salvage treatment group. VR was observed in $94.4 \%$ (34/36) of patients in the presence of ETV+ADV salvage treatment group and in $78.4 \%$ (29/37) of the patients in the absence of ETV+ADV salvage treatment group. A higher proportion of patients in the presence of ETV+ADV salvage treatment group achieved a VR at 12 months (73.3\% vs. $51.6 \%$ ) and 24 months $(93.8 \%$ vs. $80.4 \%)$ than that of patients in the absence of ETV+ADV salvage treatment group (log-rank $P=0.019$ ); however, the former subgroup had lower baseline HBV DNA values (mean, $3.21 \pm 1.22$ vs. mean, $5.08 \pm 2.14 \log _{10} \mathrm{IU} / \mathrm{mL}$ for the presence and absence of ETV+ADV salvage treatment groups, respectively).

\section{Predictors of VR in Patients Treated with TDF}

To define whether there were any differences in the rates of $V R$ according to the clinical and virological factors (such as genotypic resistance profile, the presence of ETV+ADV salvage treatment or TDF monotherapy vs. combination therapy with LAM or ETV), VR rates according to these variables were compared using a logistic regression analysis (Table 4). Univariate analysis showed that lower baseline HBV DNA levels and the presence of ETV+ADV salvage treatment prior to TDF based rescue therapy were significantly associated with the VR rates. In multivariate analysis, absolute HBV DNA level at the start of TDF rescue treatment was the only significant predictor for VR ( $\mathrm{HR}, 0.723 ; 95 \% \mathrm{Cl}, 0.627$ $0.834 ; P<0.001)$.

To determine whether an optimal cut-off level of HBV DNA at baseline of TDF treatment could predict VR, we analyzed the HBV DNA cut-off values could provide maximal predictive efficacy. Our results revealed that the best cut-off value of HBV DNA level at baseline for the prediction of VR was $5.61 \log _{10} \mathrm{IU} / \mathrm{mL}$, with a sensitivity and specificity of $80.6 \%$ and $70.0 \%$, respectively. Using cut-off value of HBV DNA at baseline, the cumulative VR rates in patients with HBV DNA levels $\geq 5.61 \log _{10} \mathrm{IU} / \mathrm{mL}(n=19)$ and those with HBV DNA levels $<5.61 \log _{10} \mathrm{IU} / \mathrm{mL}(n=54)$ were $22.7 \%$ and $77.9 \%$ at 12 months and $73.2 \%$ and $98.2 \%$ at 24 months, respectively (Fig. 3; log-rank $P<0.001$ ).

\section{Safety}

No clinically significant AEs were observed during the TDFbased treatment. Mean creatinine level and estimated glomerular filtration rate did not change during the treatment period. Two patients $(2.7 \%)$ had an increase in serum creatinine. However, no patient had an increase in serum creatinine of $>0.5 \mathrm{mg} / \mathrm{dL}$ above the baseline value. Mild hypophosphatemia (a serum phosphorus level of $<2.7 \mathrm{mg} / \mathrm{dL}$ ) was detected in one patient without change in serum creatinine. HCC occurrence was not detected in any pa- 
Hee-Jeong Jeon, et al. TDF in CHB patients with LAM and ETV resistance

Table 4. Univariate and multivariate analyses of the predictive factors for virologic response during TDF-based rescue therapy

\begin{tabular}{|c|c|c|c|c|c|c|}
\hline \multirow{2}{*}{ Variables } & \multicolumn{3}{|c|}{ Univariate } & \multicolumn{3}{|c|}{ Multivariate } \\
\hline & HR & $95 \% \mathrm{Cl}$ & $P$-value & HR & $95 \% \mathrm{Cl}$ & $P$-value \\
\hline Age (years) & 0.961 & $0.911-1.013$ & 0.141 & & & \\
\hline Gender (male gender) & 0.923 & $0.303-2.814$ & 0.888 & & & \\
\hline Diagnosis (CH/LC) & 1.896 & $0.706-5.092$ & 0.204 & & & \\
\hline Duration of TDF therapy & 0.948 & $0.813-1.106$ & 0.499 & & & \\
\hline AST & 0.998 & $0.987-1.008$ & 0.662 & & & \\
\hline ALT & 0.997 & $0.987-1.007$ & 0.507 & & & \\
\hline HBeAg positivity & 1.229 & $0.282-5.363$ & 0.784 & & & \\
\hline Pretreatment HBV DNA level & 0.699 & $0.547-0.893$ & 0.004 & 0.723 & $0.627-0.834$ & $<0.001$ \\
\hline ETV mutation profiles & 0.898 & $0.164-4.921$ & 0.901 & & & \\
\hline Presence of ETV/ADV before TDF therapy & 0.593 & $0.356-0.988$ & 0.045 & 0.611 & $0.201-1.866$ & 0.387 \\
\hline $\begin{array}{l}\text { Rescue therapy regimen (TDF monotherapy vs. TDF } \\
\text { combination therapy) }\end{array}$ & 1.052 & $0.395-2.805$ & 0.919 & & & \\
\hline
\end{tabular}

TDF, tenofovir disoproxil fumarate; HR, hazard ratio; $\mathrm{Cl}$, confidence interval; CH, chronic hepatitis; LC, liver cirrhosis; AST, aspartate aminotransferase; ALT, alanine aminotransferase; HBeAg, hepatitis B e antigen; HBV, hepatitis B virus; ETV, entecavir; ADV, adefovir dipivoxil; LAM, lamivudine.

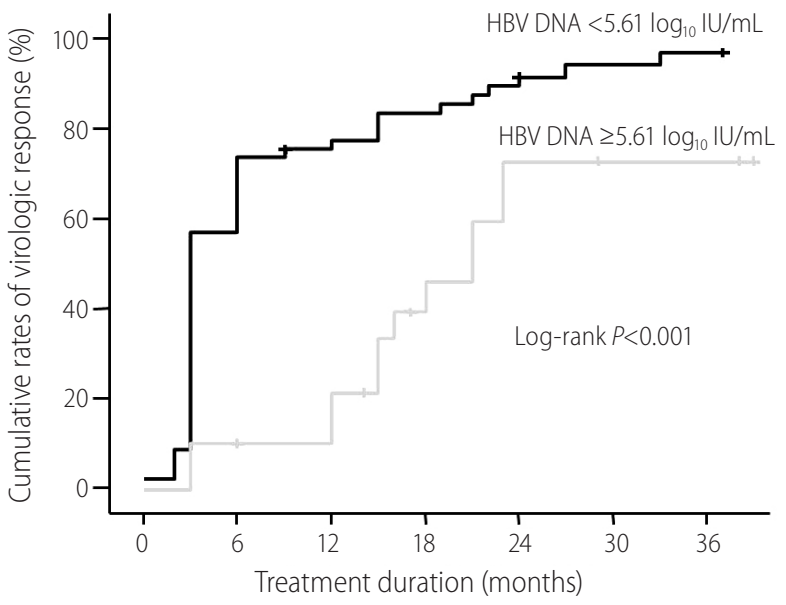

Figure 3. Cumulative rates of VR according to pretreatment HBV DNA level. The cumulative VR rates in patients with HBV DNA level $\geq 5.61 \log _{10}$ $\mathrm{IU} / \mathrm{mL}(\mathrm{n}=19)$ and those with HBV DNA level $<5.61 \log _{10} \mathrm{IU} / \mathrm{mL}(\mathrm{n}=54)$ were $22.7 \%$ and $77.9 \%$ at 12 months and $73.2 \%$ and $98.2 \%$ at 24 months, respectively. VR, virologic response; $H B V$, hepatitis $B$ virus.

tient during the follow-up period.

\section{DISCUSSION}

In our study, the long-term TDF based rescue therapy from community clinic-based settings demonstrated strong viral suppression in patients who had failed in prior treatment with ETV and
LAM. We also found that TDF monotherapy was as effective as the combination therapy of TDF+ETV or TDF+LAM for viral suppression in CHB patients who showed genotypic resistance to both LAM and ETV. Moreover, multivariate analysis revealed that the HBV DNA level at the start of TDF rescue therapy was the only independent predictor of subsequent VR.

Unfortunately, single ETV salvage therapy as one of the treatment options for CHB patients with LAM resistance was not satisfactory." In addition, sequential monotherapy could promote the selection of multi-drug resistant (MDR) HBV strains, especially when patients are treated sequentially with drugs with overlapping resistance profiles, such as with LMV followed by ETV. ${ }^{16,23,24}$ TDF has been shown to be active against ETV-resistant HBV in vitro. ${ }^{16}$ Therefore, it makes sense intuitively to utilize TDF as a rescue therapy for patients with suboptimal response or VBT during ETV therapy. Previously, several small retrospective studies demonstrated the efficacy of TDF based rescue therapy in CHB patients infected with HBV resistant to both LAM and ETV. ${ }^{17-20} \mathrm{~A}$ recent randomized controlled and multicenter prospective study showed that TDF mono-rescue therapy for 48 weeks induced a virologic response comparable to that of TDF and ETV combination therapy in patients infected with ETV-resistant HBV. ${ }^{21}$ In another study, the TDF group also showed a high virologic response rate comparable to that of the TDF+ETV group, but the duration of follow-up was relatively short (a median 18 months). ${ }^{22}$ In the present study, the median duration of TDF based rescue therapy was 
37 months. During TDF based rescue therapy, VR was achieved in 63 patients (86.3\%). The cumulative rates of VR at 12 and 24 months were $63.3 \%$ and $87.1 \%$, respectively. Results in this study were in consistent with previous studies conducted in CHB patients with LAM and ETV resistance. ${ }^{17-22}$ Taken together, these data suggest that TDF based rescue therapy is an effective option for CHB patients with treatment failure to LAM and ETV sequential therapy.

We evaluated the efficacy of TDF+ETV or TDF+LAM combination therapy compared to that of TDF monotherapy in CHB patients who developed resistance to LAM and ETV. In our study, 42 patients received TDF+ETV combination therapy, 19 patients received TDF+LAM combination therapy, and 12 received TDF monotherapy. The rates of VR among the three groups (TDF monotherapy, TDF+LAM, and TDF+ETV) were not statistically different (log-rank $P=0.200)$ at 12 months $(59.3 \%, 78.9 \%$, and 51.8 $\%$, respectively) or at 24 months $(88.4 \%, 94.7 \%$, and $84.2 \%$, respectively). In addition, treatment efficacy of TDF based combination or TDF monotherapy was not statistically different on pre-existing HBV resistant strains or exposure to other antiviral agents (ETV+ADV salvage treatment prior to TDF based rescue therapy). Thus, our studies confirmed that, compared to TDF monotherapy, TDF combination therapy did not provide any additional suppressive effect for CHB patients with resistance to LAM and ETV, which is in consistent with a few results previously reported. 17,21,22 However, a systemically designed trial with a large number of patients is needed to evaluate the efficacy of antiviral treatment strategy in CHB patients with multidrug resistance.

Many pretreatment variables have been investigated to identify predictive factors for the selection of patients most likely to respond to antiviral therapy. ${ }^{25-28}$ Among the pretreatment factors, lower baseline HBV DNA levels and the presence of ETV+ADV salvage treatment prior to TDF based rescue therapy were significantly associated with VR in the univariate analysis. Upon evaluating according to the presence or absence of ETV+ADV salvage treatment prior to TDF based rescue therapy, VR rates in the presence of ETV+ADV salvage treatment group were significantly higher than those in the absence of ETV+ADV salvage treatment prior to TDF based rescue therapy group, which might be associated with the significantly lower baseline HBV values in the group who had received ETV+ADV salvage treatment. In the multivariate analysis, only lower baseline HBV DNA level remained an independent predictor of VR. In our study, the cumulative VR rates in patients with HBV DNA levels $\geq 5.61 \log _{10} \mathrm{IU} / \mathrm{mL}(\mathrm{n}=19)$ and those with HBV DNA levels $<5.61 \log _{10} \mathrm{IU} / \mathrm{mL}(\mathrm{n}=54)$ were $22.7 \%$ and $77.9 \%$ at 12 months and $73.2 \%$ and $98.2 \%$ at 24 months, respectively. This observation is in agreement with two previous studies in CHB patients with resistance to both LAM and ETV. ${ }^{21,22}$ Switching to TDF during the early stage of ETV resistance was significantly more effective in suppressing HBV DNA than delayed rescue therapy after HBV DNA increased to higher levels.

This study had several limitations. First, a retrospective study design was used, in spite of a long-term follow-up period. Second, TDF-resistant mutational analysis was not performed in patients with continued viremia or who experienced VBT during TDF rescue therapy due to lack of TDF mutation test in our institution at that study time. Third, patient compliance to these long term treatment regimens may be poorer and less controlled than compliance to short term strictly monitored treatments in clinical trials. Despite these shortcomings, our results may be valuable to demonstrate the antiviral efficacy and safety in patients with LAM and ETV resistant HBV treated with TDF based regimens. These results may be helpful for clinicians' actual practical care of these difficult-to-treat HBV patients. To verify the efficacy and safety of TDF based rescue regimen, further large cohort studies with a long follow-up duration are warranted.

In conclusion, in CHB patients with resistance to LAM and ETV, TDF based rescue therapy was well tolerated without significant adverse events such as renal toxicity. TDF monotherapy was as effective as the combination therapy of TDF+LAM or TDF+ETV in maintaining viral suppression in CHB patients with LAM- and ETV-resistance. Therefore, add-on therapy with LAM or ETV appeared to be unnecessary, because it did not provide further benefit over TDF alone regarding VR. Moreover, multivariate analysis revealed that HBV DNA level at the start of TDF rescue therapy was the only predictor of subsequent VR. This observation suggests that switching to TDF should be done as early as genotypic resistance is detected so that we can maximize the efficacy of treatment in $\mathrm{CHB}$ patients with $\mathrm{CHB}$ with resistance to ETV.

\section{Authors' contribution}

Conception and design the work: Neung Hwa Park and Jung Woo Shin Data collection: Hee-Jeong Jeon, Yujin Yang, Jin-Hee Noh, Jae-

Sung Ahn, Hyung Rae Kim, and Jae Ho Lee Data analysis and interpretation: Seok Won Jung and Jung Woo Shin Drafting the article: Hee-Jeong Jeon and Neung Hwa Park Critical revision of the article: Seok Won Jung and Neung Hwa Park Final approval of the manuscript: All authors

\section{Financial support}

This work was supported by Priority Research Center Program 
Hee-Jeong Jeon, et al.

TDF in CHB patients with LAM and ETV resistance

through the National Research Foundation of Korea (NRF) funded by the Ministry of Education, Science, and Technology (20090094050).

\section{Conflicts of Interest}

The authors have no conflicts to disclose.

\section{REFERENCES}

1. Triolo M, Della Corte C, Colombo M. Impact of HBV therapy on the incidence of hepatocellular carcinoma. Liver Int 2014;34 Suppl 1:139-145

2. Chen CJ, Yang HI, Su J, Jen CL, You SL, Lu SN, et al. Risk of hepatocellular carcinoma across a biological gradient of serum hepatitis $B$ virus DNA level. JAMA 2006;295:65-73.

3. Iloeje UH, Yang HI, Su J, Jen CL, You SL, Chen CJ, et al. Predicting cirrhosis risk based on the level of circulating hepatitis $B$ viral load. Gastroenterology 2006;130:678-686.

4. Chang TT, Liaw YF, Wu SS, Schiff E, Han KH, Lai CL, et al. Long-term entecavir therapy results in the reversal of fibrosis/cirrhosis and continued histological improvement in patients with chronic hepatitis B. Hepatology 2010;52:886-893.

5. Marcellin P, Gane E, Buti M, Afdhal N, Sievert W, Jacobson IM, et al. Regression of cirrhosis during treatment with tenofovir disoproxil fumarate for chronic hepatitis B: a 5-year open-label follow-up study. Lancet 2013;381:468-475.

6. Hosaka T, Suzuki F, Kobayashi M, Seko Y, Kawamura Y, Sezaki H, et al. Long-term entecavir treatment reduces hepatocellular carcinoma incidence in patients with hepatitis B virus infection. Hepatology 2013;58:98-107.

7. Liaw YF, Sung JJ, Chow WC, Farrell G, Lee CZ, Yuen H, et al. Lamivudine for patients with chronic hepatitis $B$ and advanced liver disease. N Engl J Med 2004;351:1521-1531.

8. Lok AS, Lai CL, Leung N, Yao GB, Cui ZY, Schiff ER, et al. Long-term safety of lamivudine treatment in patients with chronic hepatitis $B$. Gastroenterology 2003;125:1714-1722.

9. Liaw YF, Leung N, Kao JH, Piratvisuth T, Gane E, Han KH, et al. Asian-pacific consensus statement on the management of chronic hepatitis B: a 2008 update. Hepatol Int 2008;2:263-283.

10. Lok AS, McMahon BJ. Chronic hepatitis B. Hepatology 2007:45:507539.

11. Tenney DJ, Rose RE, Baldick CJ, Pokornowski KA, Eggers BJ, Fang $J$, et al. Long-term monitoring shows hepatitis b virus resistance to entecavir in nucleoside-naive patients is rare through 5 years of therapy. Hepatology 2009;49:1503-1514.

12. Yuen MF, Sablon E, Hui CK, Yuan HJ, Decraemer H, Lai CL. Factors associated with hepatitis $B$ virus DNA breakthrough in patients receiving prolonged lamivudine therapy. Hepatology 2001;34(4 Pt
1):785-791.

13. Ghany MG, Doo EC. Antiviral resistance and hepatitis B therapy. Hepatology 2009;49(5 Suppl):S174-S184.

14. European Association For The Study Of The Liver. Easl clinical practice guidelines: Management of chronic hepatitis B virus infection. J Hepatol 2012;57:167-185.

15. Lok AS, McMahon BJ. Chronic hepatitis B: update 2009. Hepatology 2009;50:661-662.

16. Villet S, Ollivet A, Pichoud C, Barraud L, Villeneuve JP, Trépo C, et al. Stepwise process for the development of entecavir resistance in a chronic hepatitis B virus infected patient. J Hepatol 2007;46:531-538.

17. Kim YJ, Sinn DH, Gwak GY, Choi MS, Koh KC, Paik SW, et al. Tenofovir rescue therapy for chronic hepatitis B patients after multiple treatment failures. World J Gastroenterol 2012;18:6996-7002.

18. Yip B, Chaung K, Wong CR, Trinh HN, Nguyen HA, Ahmed A, et al. Tenofovir monotherapy and tenofovir plus entecavir combination as rescue therapy for entecavir partial responders. Dig Dis Sci 2012;57:3011-3016.

19. Wong CR, Trinh HN, Yip B, Nguyen HA, Garcia RT, Ahmed A, et al. High rate of complete viral suppression with combination therapy in patients with chronic hepatitis B and prior treatment failure. J Clin Gastroenterol 2011;45:900-905.

20. Petersen J, Ratziu V, Buti M, Janssen HL, Brown A, Lampertico P, et al. Entecavir plus tenofovir combination as rescue therapy in pretreated chronic hepatitis B patients: an international multicenter cohort study. J Hepatol 2012;56:520-526.

21. Lim YS, Byun KS, Yoo BC, Kwon SY, Kim YJ, An J, et al. Tenofovir monotherapy versus tenofovir and entecavir combination therapy in patients with entecavir-resistant chronic hepatitis B with multiple drug failure: results of a randomised trial. Gut 2016;65:852-860.

22. Lee S, Ahn SH, Jung KS, Kim DY, Kim BK, Kim SU, et al. Tenofovir versus tenofovir plus entecavir for chronic hepatitis B with lamivudine resistance and entecavir resistance. J Viral Hepat 2017;24:141-147.

23. Yim HJ, Hussain M, Liu Y, Wong SN, Fung SK, Lok AS. Evolution of multi-drug resistant hepatitis $B$ virus during sequential therapy. Hepatology 2006;44:703-712.

24. Kim BG, Jung SW, Kim EH, Kim JH, Park JH, Sung SJ, et al. Tenofovirbased rescue therapy for chronic hepatitis B patients who had failed treatment with lamivudine, adefovir, and entecavir. J Gastroenterol Hepatol 2015;30:1514-1521.

25. Zoutendijk R, Reijnders JG, Brown A, Zoulim F, Mutimer D, Deterding $K$, et al. Entecavir treatment for chronic hepatitis $B$ : adaptation is not needed for the majority of naïve patients with a partial virological response. Hepatology 2011;54:443-451.

26. Yuen MF, Seto WK, Fung J, Wong DK, Yuen JC, Lai CL. Three years of continuous entecavir therapy in treatment-naïve chronic hepatitis B patients: VIRAL suppression, viral resistance, and clinical safety. Am J Gastroenterol 2011;106:1264-1271. 
27. Ono A, Suzuki F, Kawamura Y, Sezaki H, Hosaka T, Akuta N, et al. Long-term continuous entecavir therapy in nucleos(t)ide-naïve chronic hepatitis B patients. J Hepatol 2012;57:508-514.

28. Shin JW, Jung SW, Park BR, Kim CJ, Eum JB, Kim BG, et al. Pre- diction of response to entecavir therapy in patients with $\mathrm{HBeAg}$ positive chronic hepatitis B based on on-treatment $\mathrm{HBsAg}, \mathrm{HBeAg}$ and HBV DNA levels. J Viral Hepat 2012;19:724-731. 